\title{
Functional and usability assessment of a robotic exoskeleton arm to support activities of daily life Emilia Ambrosini $\dagger \|^{*}$, Simona Ferrante $\dagger \|$, Mauro Rossinił, Franco Moltenił, Margit Gföhler§, Werner Reichenfelser§, Alexander Duschau-Wicke $\Phi$, Giancarlo Ferrigno $\dagger$ and Alessandra Pedrocchi $\dagger$
}

\author{
$\dagger$ NeuroEngineering and Medical Robotics Laboratory, NearLab, Department of Electronics, \\ Information, and Bioengineering, Politecnico di Milano, Milan, Italy \\ $\ddagger$ Valduce Hospital, Villa Beretta, Rehabilitation Centre, Costa Masnaga, Lecco, Italy \\ $\S$ Research Group for Machine Design and Rehabilitation Engineering (E 307-3), Technische \\ Universität Wien, Vienna, Austria \\ \Hocoma AG, Volketswil, Switzerland
}

(Accepted June 19, 2014. First published online: July 22, 2014)

\begin{abstract}
SUMMARY
An assistive device for upper limb support was developed and evaluated in terms of usability, user satisfaction and motor performance on six end-users affected by neuro-motor disorders (three spinal cord injury; one multiple sclerosis; two Friedreich's ataxia). The system consisted of a lightweight 3-degrees-of-freedom robotic exoskeleton arm for weight relief, equipped with electromagnetic brakes. Users could autonomously control the brakes using a USB-button or residual electromyogram activations. The system functionally supported all of the potential users in performing reaching and drinking tasks. For three of them, time, smoothness, straightness and repeatability were also comparable to healthy subjects. An overall high level of usability (system usability score, median value of 90/100) and user satisfaction (Tele-healthcare Satisfaction Questionnaire - Wearable Technology, median value of 104/120) were obtained for all subjects.
\end{abstract}

KEYWORD: Assistive device; Exoskeleton; Neuro-motor disorders; Upper limb; Daily life activities.

\section{Introduction}

An assistive device (AD) is defined as any product especially designed to prevent, compensate, relieve or neutralize impairments, activity limitations and participation restrictions. Several reasons foster the society to the development of ADs, such as the increase in the number of people with disabilities and the greater need for individual mobility and autonomy due to a more centralized organization of the society. The design of an AD has to satisfy primarily the end-user needs. ${ }^{1}$ People with severe neuro-motor disabilities have the main expectation of recovering a direct and easy interaction with objects of daily life in order to regain independence and self-esteem.

Most of the ADs are robotic systems aimed at increasing the independence of people with disabilities providing physical assistance for both upper and lower limbs. ${ }^{2}$ Concerning the assistance for the upper limb, the existing robotic ADs include external manipulators ${ }^{3-5}$ and passive $^{6-8}$ or powered exoskeletons. ${ }^{9}$ Among the external manipulators, the Bridgit and the JACO robotic arms ${ }^{1}$ are mounted on the user's wheelchair or on a rail-system and assist the user in the execution of numerous activities of daily life such as self-feeding, self-care or household activities without requiring any

\footnotetext{
* Corresponding author. E-mail: emilia.ambrosini@ polimi.it

"Emilia Ambrosini and Simona Ferrante contributed equally to this work as joint first co-authors.

1 www.focalmeditech.nl/en/products/personal-robotics.html
} 
voluntary upper limb movements. ${ }^{3}$ All of these systems are normally not directly connected to the user's arm and completely surrogate the user's action. Passive exoskeletons instead are used to reduce or eliminate the effect of gravity by using spring and damper elements and allow the users to perform functional tasks with their weak residual muscle effort. ${ }^{6,7}$ An alternative solution to provide gravity compensation consists of the application of electro-rheological fluids as controlled resistive torque elements that allow coordinated joint damping or even locking and unlocking of the degrees of freedom (DOFs). ${ }^{10}$ This solution usually requires cumbersome systems actuated by external motors. A number of powered exoskeletons for the upper extremity have been also developed. In these devices, joint actuation is controlled by means of external drives (e.g. electrical motors) and predefined movements are offered by the system, so as that the user loses the control of his/her own actions. Most advanced instrumented and/or powered exoskeleton systems, such as the MIT-MANUS, ${ }^{11}$ the Armeo ${ }^{\circledR}$ Power (Hocoma, Switzerland) and the ARMin, ${ }^{9,12}$ have been developed and mainly used for stationary rehabilitation exercises in a clinical environment. These systems are heavy, cumbersome and expensive, being then not suited for being used at home. Different studies have been recently carried out with the common aim to enrich powered exoskeletons with human-robot interfaces capable of understanding the intention of the user and reacting promptly to provide the required assistance. Kiguchi and Hayashi ${ }^{13}$ proposed an electromyogram (EMG)-based impedance control method for an upper-limb power-assist exoskeleton. Their system demonstrated to be effective for rehabilitative purposes but the number of EMG electrodes used and the inadequate usability outside a specialized rehabilitation centre limit its applicability as an $\mathrm{AD}$. In order to combine ease of use and effectiveness in assisting movement, a simplified EMG-based assistive robotic system for elbow assistance has been proposed. ${ }^{14}$ Experiments on healthy volunteers showed that a full control of the movement was kept while reducing the required effort. Further investigation is needed to prove the effectiveness of this system on people with reduced motor capabilities.

In order to quantitatively evaluate the performance while executing robot-assisted movements, the subjects are usually asked to perform repetitive, predefined arm movements in the robot workspace. Different measures of performance based on signals extracted from the sensors embedded in the robots have been defined. Some examples are the range of motion, the movement duration, the linearity, symmetry, smoothness and repeatability of the movement, or the force produced in the movement direction. ${ }^{15-17}$ These measures are used in rehabilitation robotics to monitor changes in motor control of the affected limb during the course of the intervention. ${ }^{17}$ In assistive robotics, a functional assessment of the support offered by the system during daily life activities is usually provided. This assessment might be in the form of a descriptive report of the user's experience, ${ }^{7,8}$ a score assigned by experts (from $0=$ unsuccessful support, to $2=$ completely functional) ${ }^{18}$ or a visual analogue scale to assess the users' mood, motivation and general satisfaction. ${ }^{19}$ As far as we know, no studies have been evaluated ADs exploiting the robot as a measuring tool.

Bearing in mind that the main user requirements for ADs are wearability, lightweight, usability and the capability to understand the user's intention without imposing predefined movements, a robotic system to support upper limb daily life activities was developed during the European Project MUNDUS ${ }^{2} .{ }^{18,20}$ A modular system that can be adapted to different levels of disability and/or to the progression of the disease was proposed. The present study aimed at quantitatively evaluating the configuration of the MUNDUS system suitable for users with some residual motor capabilities. This configuration consisted of a spring-loaded robotic exoskeleton arm for gravity compensation provided with electromagnetic brakes. Usability, user satisfaction and performance evaluation metrics based on joint angle data have been assessed on six people affected by neuro-motor disorders. A group of healthy volunteers was also involved in the trials for comparison.

\section{Methods}

\subsection{Apparatus}

The apparatus is shown in Fig. 1 and consisted of a lightweight passive exoskeleton arm with 3 DOFs: shoulder elevation in the sagittal plane, shoulder rotation in the horizontal plane

${ }^{2}$ www.mundus-project.eu 


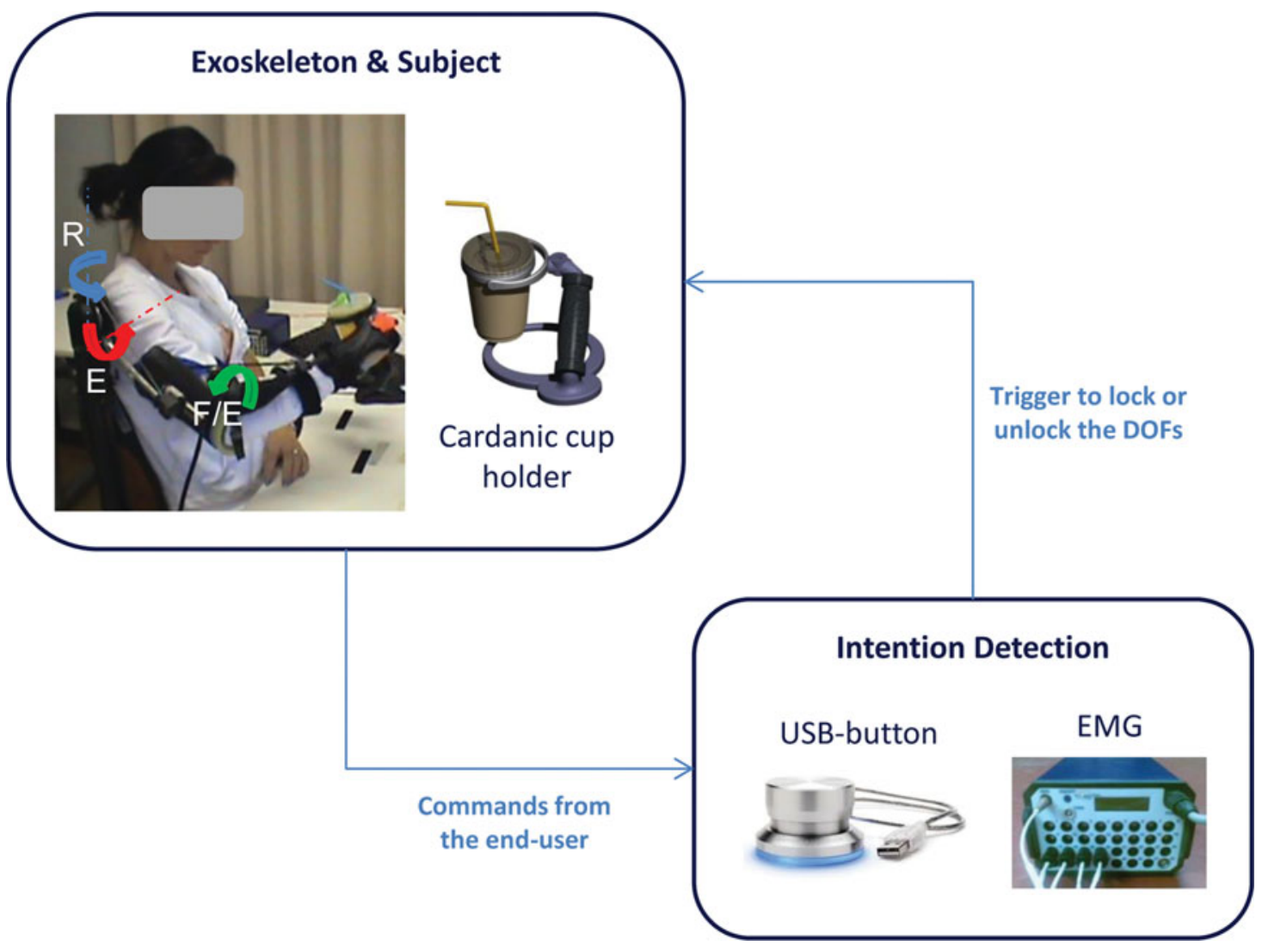

Fig. 1. (Colour online) Experimental setup. The 3 degrees of freedom are shown: shoulder elevation in the sagittal plane (red arrow; E), shoulder rotation in the horizontal plane (blue arrow; R), and elbow flexion/extension (green arrow; F/E).

and elbow flexion/extension. ${ }^{20}$ Humeral rotation was fixed, and forearm prono/supination, wrist flexion/extension and wrist deviation were mechanically fixed at subject-specific positions using a rigid wrist orthosis. A spring mechanism to provide an adjustable weight support was included in the mechanical shoulder joint. By changing the spring pretension manually, an arm weight up to $5.2 \mathrm{~kg}$ could be compensated from $0 \%$ to $100 \%$. Electromagnetic DC brakes could lock each DOF. Encoders to measure the angles at the 3 DOFs (sampling frequency of $50 \mathrm{~Hz}$ ) were also included. The exoskeleton, which was characterized by a total weight of $2.2 \mathrm{~kg}$, could be mounted on a wheelchair, as shown in Fig. 1, or used as a mobile system together with a body harness. Up to now only a right-arm prototype of the exoskeleton was developed.

To compensate for the fixed orientation of the hand vector, a cardanic cup holder (Fig. 1) was also designed. This holder kept the cup vertical independently from the hand position. For the interaction with other objects of daily life, such as comb or brush, a similar auxiliary tool was developed to keep the object at the desired orientation angle.

The user could independently lock or unlock the DOFs by alternatively using one of the following solutions: a USB-button controlled by the contralateral hand or the EMG signal of a muscle of the contralateral arm which the user can still voluntarily control. In the case of the second solution, $\mathrm{Ag} / \mathrm{AgCl}$ pre-gelled self-adhesive electrodes were placed over the target muscle and an EMG amplifier (Porti $32^{\mathrm{TM}}$, TMS International BV, Enschede, The Netherlands) was used to acquire the EMG signal at $2048 \mathrm{~Hz}$. An ad hoc real-time algorithm was developed to detect the user's intention: when a sequence of three consecutive contractions, lasting at least $1 \mathrm{~s}$ each, within a time interval of $10 \mathrm{~s}$, was recognized by the algorithm, a trigger was sent to the system and provoked the locking or unlocking of the DOFs, depending on their current status. ${ }^{22}$ 
Table I. Clinical and demographic characteristics of the end-users.

\begin{tabular}{|c|c|c|c|c|c|c|c|c|c|c|c|c|}
\hline \multirow[b]{2}{*}{ Subject } & \multirow{2}{*}{$\begin{array}{l}\text { Age } \\
\text { (years) }\end{array}$} & \multirow[b]{2}{*}{ Sex } & \multirow[b]{2}{*}{ Pathology } & \multirow{2}{*}{$\begin{array}{l}\text { Time since } \\
\text { injury } \\
\text { (years) }\end{array}$} & \multirow{2}{*}{$\begin{array}{l}\text { Motricity } \\
\text { Index } \\
(0-100)\end{array}$} & \multicolumn{4}{|c|}{$\begin{array}{l}\text { Barthel Index (single item: } \\
\text { 0-2; total: 0-20) }\end{array}$} & \multirow{2}{*}{$\begin{array}{l}\text { Trigger } \\
\text { modality }\end{array}$} & \multicolumn{2}{|c|}{$\begin{array}{c}\text { Anthropometric } \\
\text { measures }(\mathrm{m})\end{array}$} \\
\hline & & & & & & Feeding & Grooming & Dressing & Total & & Arm & Forearm \\
\hline P1 & 27 & M & SCI & 1 & 84 & 1 & 0 & 0 & 3 & Button & 0.28 & 0.24 \\
\hline $\mathrm{P} 2$ & 51 & M & FRDA & 27 & 71 & 1 & 0 & 1 & 7 & EMG & 0.31 & 0.28 \\
\hline P3 & 70 & M & SCI & 1 & 39 & 0 & 0 & 1 & 5 & EMG & 0.33 & 0.28 \\
\hline $\mathrm{P} 4$ & 45 & M & MS & 9 & 77 & 1 & 0 & 1 & 4 & Button & 0.32 & 0.26 \\
\hline P5 & 61 & M & SCI & 2 & 58 & 1 & 1 & 1 & 6 & Button & 0.28 & 0.28 \\
\hline P6 & 29 & $\mathrm{~F}$ & FRDA & 7 & 76 & 1 & 0 & 1 & 4 & EMG & 0.28 & 0.32 \\
\hline
\end{tabular}

$\mathrm{SCI}=$ spinal cord injury; MS = multiple sclerosis; FRDA = Friedreich's ataxia.

Table II. Demographic characteristics of the healthy volunteers.

\begin{tabular}{lcccc}
\hline & & & \multicolumn{2}{c}{ Anthropometric measures (m) } \\
\cline { 5 - 5 } Subject & Age (years) & Sex & Arm & Forearm \\
\hline S1 & 24 & F & 0.29 & 0.28 \\
S2 & 23 & F & 0.33 & 0.32 \\
S3 & 20 & M & 0.38 & 0.35 \\
S4 & 21 & F & 0.30 & 0.31 \\
S5 & 45 & M & 0.29 & 0.31 \\
\hline
\end{tabular}

\subsection{Participants}

Six people affected by neuro-motor disorders (three spinal cord injury (SCI); one multiple sclerosis (MS); two Friedreich's ataxia (FRDA)) were selected among potential end-users and involved in the experimental trials. Table I reports the clinical and demographic details of the participants.

Five healthy volunteers with no history of muscle weakness or neurological disorders were also involved in the study to define the normality ranges of the performance evaluation metrics based on joint angle data. Details of healthy volunteers are reported in Table II.

All of the trials were performed at the Villa Beretta Rehabilitation Centre. The study was approved by the Ethical Committee of the Valduce Hospital and each subject who agreed to participate to the study signed a written informed consent.

\subsection{Experimental protocol}

The end-users were asked to perform three experimental sessions over consecutive days. Before the beginning of the first session, the exoskeleton was adjusted by the caregiver to the anthropometric measures of each subject and the weight compensation was set by changing the spring pretension manually. The arm weight of the subjects was slightly undercompensated to favour a gravity-driven return of the arm to the rest position. Then, the solution to control the locking and unlocking of the DOFs was selected: if the user was able to control the contralateral hand, the USB-button was preferred for its simplicity; otherwise, the EMG-based modality was used. The subject was asked which muscle of the contralateral arm he/she preferred to use to control the brakes. Fast trials to assess whether the algorithm was able to effectively recognize contractions of the selected muscle were performed.

Within each session two tasks were performed: drinking with a straw and reaching the contralateral shoulder. The execution order was randomized among subjects and days. For each task, 10 repetitions were performed. Each repetition was divided into the following sub-actions:

1. Reach the target (either the mouth or the shoulder) from the rest position and then lock the DOFs;

2. Wait for at least $10 \mathrm{~s}$;

3. Unlock the DOFs and go back to the rest position. 
During the drinking task, the subject had first to reach the cup and then bring it to the mouth and vice-versa. The rest position as well as the placement of the cup on the table were not standardized among subjects but selected independently by each user.

Healthy subjects were involved in a single day session, following the same procedure used for the end-users. To control the brakes, the EMG trigger solution was used in order to provide also a validation of the algorithm.

\subsection{Data analysis and statistics}

To evaluate the motor performance during the execution of the tasks, the $x, y$ and $z$ coordinates of the wrist were reconstructed using the Robotics toolbox for Matlab ${ }^{\circledR}$, starting from the angular data acquired at the 3 DOFs of the exoskeleton. The humeral rotation was fixed, while the lengths of the arm and the forearm were defined based on the anthropometric measures of each subject. Each repetition was divided into two sub-actions: the forward and backward movements. The forward movement was defined as the movement from the rest position (for the reaching task) or the object position (for the drinking task) to the target. Analogously, the backward movement was defined as the movement from the target to the rest position (for reaching) or to the object position (for drinking). This means that for the drinking task, the planar movements for reaching the object from rest and vice-versa were not included in the analysis.

For each sub-action, the time required to accomplish the movement, the smoothness, as the ratio between the average speed and the maximal speed, and the straightness, as the distance between the initial and the final position divided by the trajectory path covered by the subject, ${ }^{22}$ were computed. Since the assumption of normal distribution was not valid in many cases, a Mann-Whitney $U$-test $(p<0.01)$ was used to compare the performance evaluation metrics achieved by each end-user during the three sessions with those obtained by the healthy subjects (control group).

To evaluate the individual repeatability for each sub-action of the two supported tasks, the coefficient of periodicity (CP) for the 3D wrist trajectory was computed. ${ }^{23,24}$ First, an upsampling or downsampling algorithm was applied to each repetition in order to obtain a uniform time-scaling. Three matrixes (one per coordinate), whose rows corresponded to the time-normalized repetitions of each sub-action, were defined. Then, single value decomposition (SVD) was applied and the CP was computed as follows:

$$
\mathrm{CP}_{i}=\frac{\sigma_{1}^{2}}{\sum_{k=1}^{N} \sigma_{k}^{2}},
$$

where $\sigma_{k}$ indicates the $k$ th eigenvalue of the SVD, $N$ is the number of the time-scaled repetitions and $i$ indicates alternatively the $x, y, z$ coordinates of the wrist. Finally, the overall CP for the 3D trajectory was defined as the mean value of the coefficients computed for each component. CP varied from 0 (no repeatability) to 1 (full repeatability).

An overall score of the motor performance achieved by each end-user was computed. For each sub-action (forward and backward movement; reaching and drinking task) and for each kinematic parameter (time, smoothness, straightness and repeatability), a score of 1 (different from the control group) or 0 (similar to the control group) was assigned. Regarding the time, the smoothness and the straightness, a score of 1 was assigned when a significant worst performance of the enduser was found with respect to the healthy subjects; concerning the repeatability, a score of 1 was assigned when the median value of the $\mathrm{CP}$ was lower than the 25th percentile of the values obtained by the healthy subjects. The overall score ranged from 0 (best performance) to 16 (worst performance).

User satisfaction with the system and usability were assessed by means of two validated instruments: the 'Tele-healthcare Satisfaction Questionnaire - Wearable Technology' (TSQ-WT; ${ }^{\circ}$ RBMF 2010) and the system usability scale (SUS; ${ }^{\circ}$ Digital Equipment Corporation). ${ }^{26}$ The TSQWT contains six subscales evaluating the benefit, usability, self-concept, privacy and loss of control, quality of life and wearing comfort of the system. Each subscale includes five questions rated on a 5-point Likert scale between 0 (I strongly disagree) and 4 (I strongly agree). The total score ranges between 0 (no satisfaction) and 120 (extreme satisfaction). The SUS includes 10 questions rated by means of a 5-point Likert scale, from 1 (I strongly disagree) to 5 (I strongly agree). The final score ranges between 0 (no satisfaction) and 100 (extreme satisfaction). 
(a)

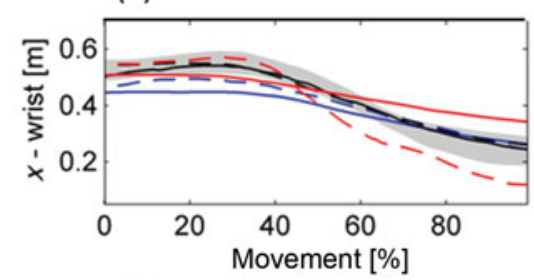

(c)

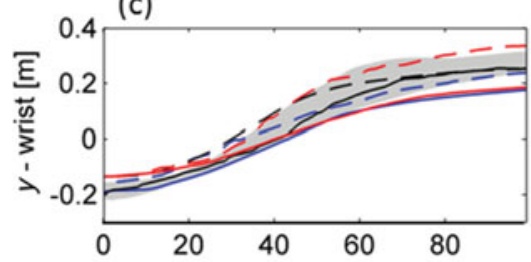

(e)

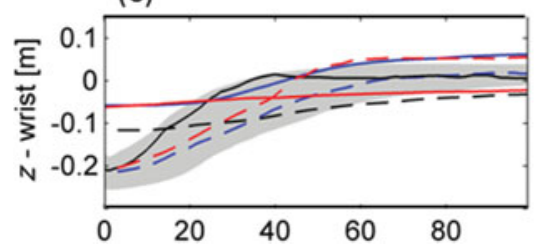

(b) Backward

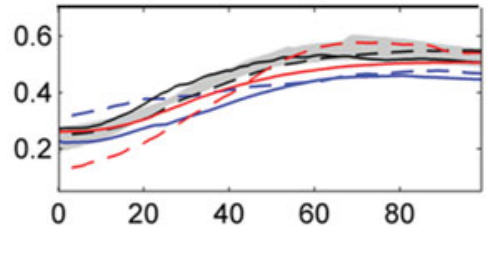

(d)

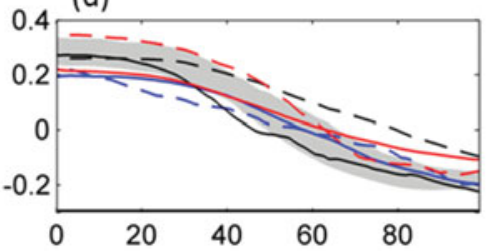

(f)

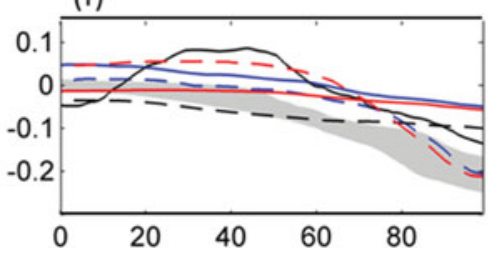

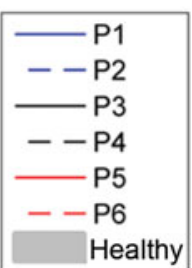

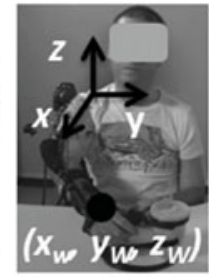

Fig. 2. (Colour online) Median trajectories of the wrist with respect to the percentage of the movement obtained by each end-user considering all of the repetitions of the reaching task. The grey area represents the range between the 25 th and the 75 th percentile of the healthy subjects. On the right, the reference frame, with the origin places in the right shoulder, is shown.

\section{Results}

All of the end-users were severely compromised in performing activities of daily life. None of them was capable of eating, combing or dressing themselves autonomously, and the total Barthel Index was always lower than $8 / 20$. Nevertheless, they were all able to accomplish drinking and reaching tasks with the support provided by the passive exoskeleton arm.

The trigger modality to control the brakes was selected according to the users' needs: three subjects controlled the USB-button with their contralateral hand, while the other three preferred to exploit the EMG activation of a muscle of the contralateral arm (see Table I). All of them used the trapezius muscle. Independently from the trigger modality, all of subjects were always able to control the brakes and none of them reported any problem or discomfort. When the USB-button was used, less than 1 $\mathrm{s}$ was enough to send a trigger to the system. However, when the EMG trigger modality was used, a mean time of $10 \mathrm{~s}$, comparable to the one of healthy volunteers, was required and no false alarms occurred.

Figures 2 and 3 show a qualitative comparison between the median trajectories of the wrist obtained by each end-user and the trajectories achieved by the healthy subjects group (grey area) during the reaching and the drinking task, respectively. In both figures, panels $(a, c, e)$ and $(b, d, f)$ report the performance achieved during the forward and backward movements, respectively. Regarding the reaching task (Fig. 2), the trajectories of the end-users along the $x$ - and $y$-axes were similar to those of the healthy subjects; the slight variability might be explained by the different anthropometric measures of each subject. A higher variability can be noticed on the wrist trajectory along the $z$-axis. This may be due to the different levels of weight compensation provided to each subject. Indeed, the compensation was manually adjusted in a range from $70 \%$ to $95 \%$ of the subjects' arm weight. Moreover, P3 was characterized by a more jerky trajectory along the vertical direction.

Table III reports the median values and the interquartile ranges of the kinematic parameters computed on the healthy subject volunteers during the two motor tasks. Figures 4 and 5 show the values of time, smoothness, straightness and $\mathrm{CP}$ obtained during the reaching and drinking tasks, respectively. During reaching (Fig. 4), four end-users (P2, P3, P4 and P6) accomplished the task significantly more slowly than the healthy group both during the forward and backward movements; the smoothness was worse than that of the control group for five out of six end-users during the 
Table III. Kinematic measures obtained by the healthy subjects group. Medial values (interquartile range) are reported.

\begin{tabular}{lcccc}
\hline & Time (s) & Smoothness & Straightness & Coefficient of periodicity \\
\hline $\begin{array}{l}\text { Reaching task } \\
\text { Forward }\end{array}$ & $1.22(0.24)$ & $0.566(0.066)$ & $0.890(0.093)$ & $0.991(0.036)$ \\
Backward & $1.27(0.33)$ & $0.594(0.103)$ & $0.849(0.077)$ & $0.967(0.034)$ \\
Drinking task & & & & \\
Forward & $1.14(0.32)$ & $0.529(0.060)$ & $0.943(0.049)$ & $0.987(0.051)$ \\
Backward & $1.26(0.58)$ & $0.592(0.104)$ & $0.901(0.039)$ & $0.956(0.043)$ \\
\hline
\end{tabular}

(a)
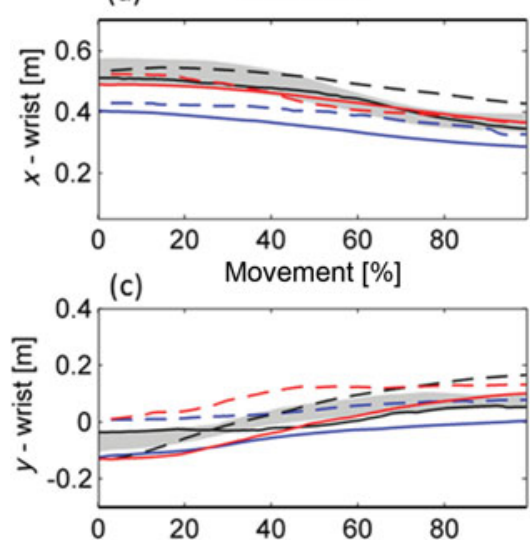

(e)

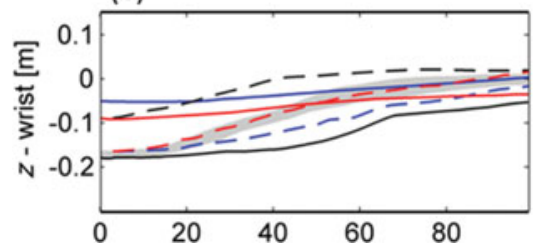

(b) Backward

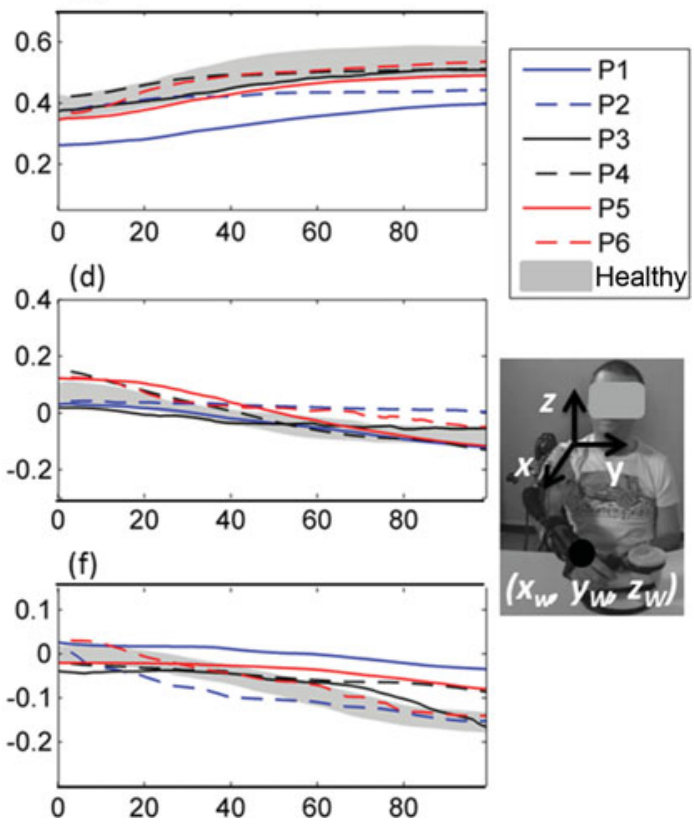

Fig. 3. (Colour online) Median trajectories of the wrist with respect to the percentage of the movement obtained by each end-user considering all of the repetitions of the drinking task. The grey area represents the range between the 25 th and the 75 th percentile of the healthy subjects. On the right, the reference frame, with the origin places in the right shoulder, is shown.

forward movement, and for only three of them during the backward movement; the straightness was significantly lower than the control group for P3 and P6 in both movements. During drinking (Fig. 5), P1, P4 and P5 showed a good motor performance; indeed no kinematic parameters resulted significantly worse than the one obtained by the control group. Instead, P2, P3 and P6 carried out a drinking task significantly slower and more jerky than the control group. The repeatability of the movement (panels (g, h) of Figs. 4 and 5) was high for all of the end-users (median value of CP always higher than 0.9). The median value of $\mathrm{CP}$ was lower than the 25th percentile of the control group in two out of four sub-actions for P2 and P3, indicating a less repeatable movement execution.

The overall score, shown in Fig. 6, summarized the motor performance achieved by each end-user in the four sub-actions. Three out of six (P1, P4 and P5) were characterized by a performance similar to the control group (overall score $<4$ ).

Results in terms of usability and user satisfaction are reported in Fig. 7. An overall high satisfaction level was obtained: the median value of the SUS was 90/100 (interquartile range 7.5) and the median value of the TSQ-WT was 104/120 (interquartile range 3.3). 


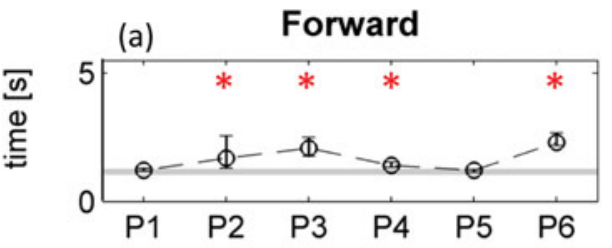

(c)

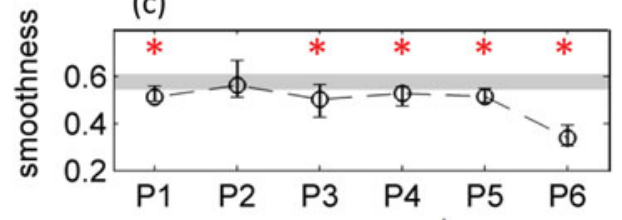

(e)

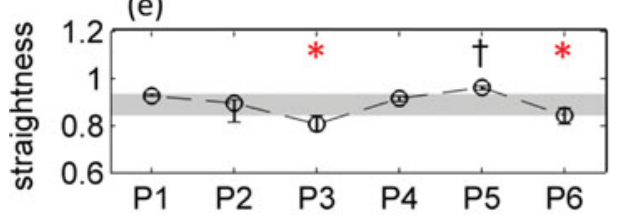

(g)

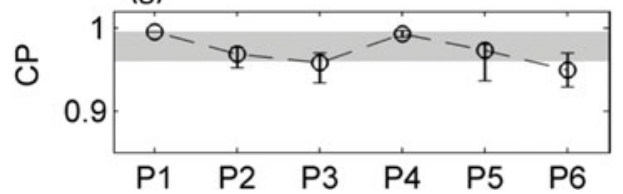

(b) Backward

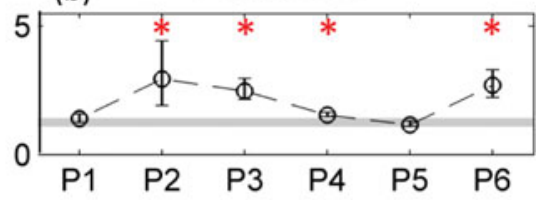

(d)
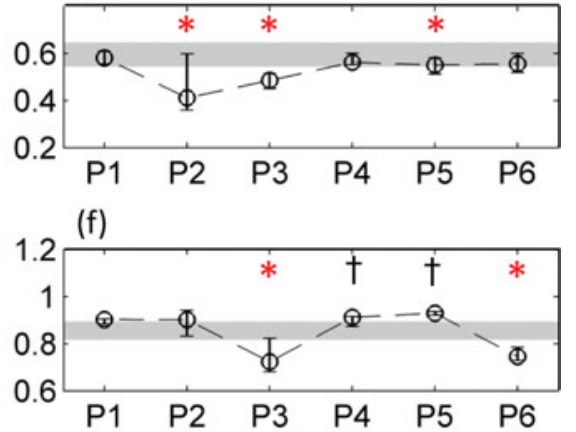

(h)

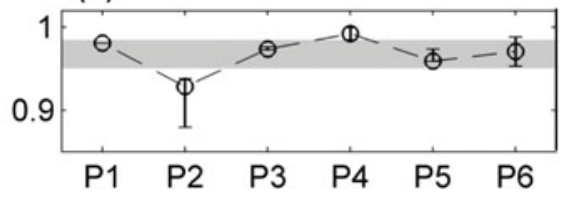

Fig. 4. (Colour online) Median values and interquartile ranges of time, smoothness, straightness and coefficient of periodicity (CP) computed on each end-user considering all of the repetitions of the reaching task. The grey area represents the range between the 25th and the 75th percentile of the healthy subjects. The asterisks indicate a significant difference $(p<0.01)$ between each end-user and the control group ${ }^{*}$ and $\dagger$ indicate an end-user's performance significantly worse or better than the control group, respectively).

\section{Discussions}

A passive robotic exoskeleton arm equipped with electromagnetic brakes was developed to support people affected by neuro-motor disorders in performing upper limb daily life activities. The system was evaluated in terms of motor performance both on six potential end-users and on a group of five healthy volunteers that were recruited to provide normality ranges. The usability of the proposed system and user satisfaction were also assessed using two validated questionnaires.

The end-users understood how to use the system from the first session. The system effectively supported them in the execution of reaching and drinking tasks, despite of their high level of disability. They obtained $100 \%$ success in locking and unlocking the DOFs using alternatively the USB-button and the EMG-based trigger modality.

A comparison in terms of motor performance between the end-users and the healthy volunteers was performed. A statistical analysis comparing each end-user over the three sessions to the control group was preferred to a group analysis, since the end-users strongly differed in terms of age, pathology and time since injury. The results of the statistical analysis highlighted that three out of six end-users (P2, P3 and P6) were characterized by a more impaired motor performance with respect to the control group during both reaching and drinking. The duration of the forward and backward movements was always significantly longer than that of the control group and in most of the sub-actions their movement was more jerky. This might be explained by the fact that $\mathrm{P} 3$ was the most impaired enduser (MI = 39, Table I), while the other two subjects (P2 and P6) suffered from FRDA, a pathology that severely affects motor coordination and movement smoothness. ${ }^{26,27} \mathrm{P} 2$ and P6 were classified as stage 5 according to the Friedreich's Ataxia Rating scale, which ranges from 0 (normal) to 6 (total disability). The proposed system might increase the independence of subjects from stage 4 upward, when the progression of the disease starts compromising the upper limb motor functions. The remaining three end-users (P1, P4 and P5) not only achieved the completion of the functional tasks, but also obtained a motor performance similar to the control group (overall score $<4 / 16$ ). 

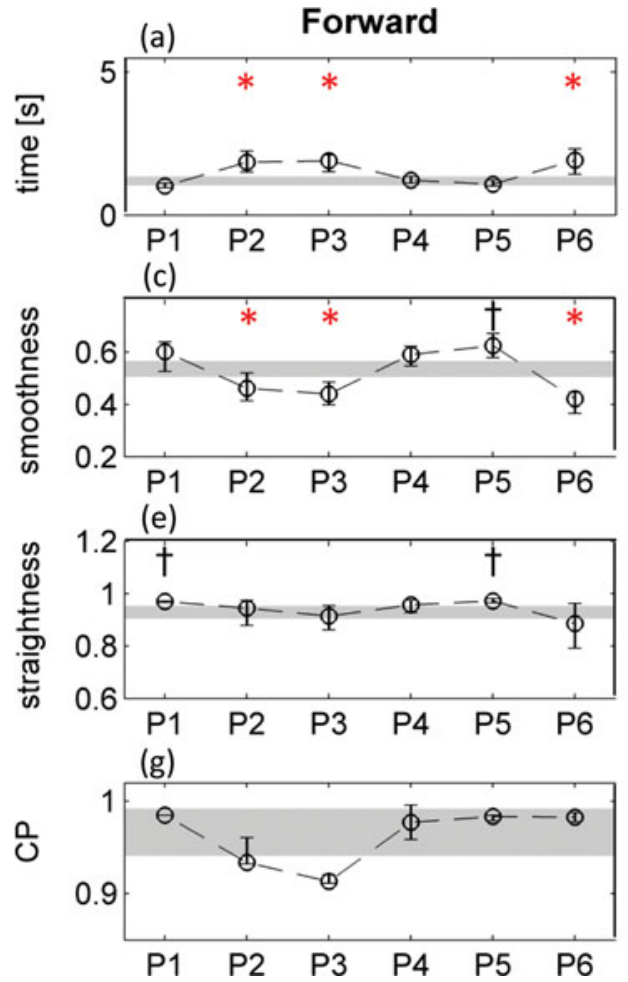

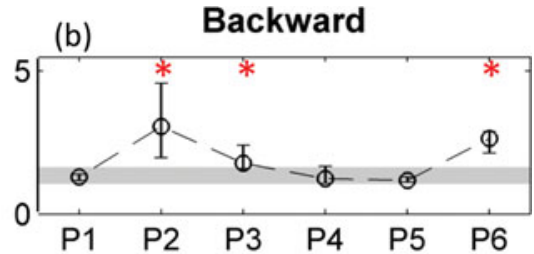

(d)
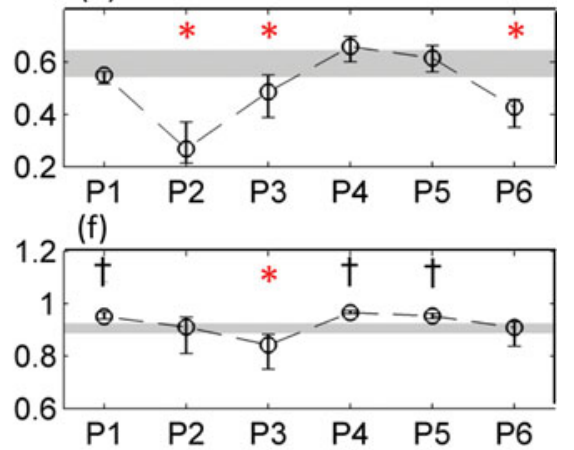

(h)

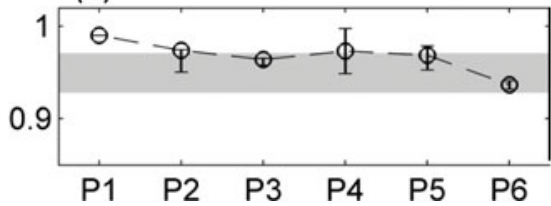

Fig. 5. (Colour online) Median values and interquartile ranges of time, smoothness, straightness and coefficient of periodicity $(\mathrm{CP})$ computed on each end-user considering all of the repetitions of the drinking task. The grey area represents the range between the 25 th and the 75 th percentile of the healthy subjects. The asterisks indicate a significant difference $(p<0.01)$ between each end-user and the control group ${ }^{*}$ and $\dagger$ indicate an end-user's performance significantly worse or better than the control group, respectively).

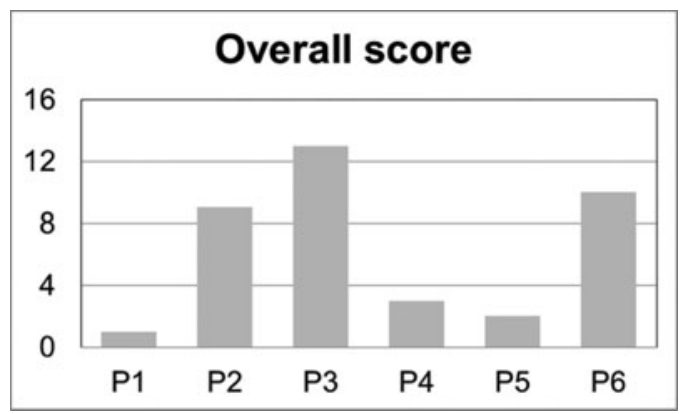

Fig. 6. Motor performance achieved by each end-user in terms of overall score.

Overall, for all of the subjects both the forward and the backward movements were highly repeatable (CP was always greater than 0.9).

All of the end-users reported the robotic exoskeleton arm to be non-cumbersome, easy to use and useful in assisting them during daily life activities. They also pointed out that it provided them with the 're-new experience' of being able to perform lost movements, improving their self-esteem and independence. They particularly liked two aspects: the possibility to perform the movement with less fatigue while preserving the control of the movement, and the possibility to maintain fixed positions without any effort thanks to the electromagnetic brakes. Furthermore, the cardanic cup holder (Fig. 1), which maintained the vertical position of the cup without requiring a fine control of the hand orientation, was considered very helpful. Some of them reported that they would have wished to use it also as a stand-alone system during their daily life. These qualitative results, derived from spontaneous reports of the users, were confirmed by the high scores obtained in the two questionnaires evaluating usability and user satisfaction (Fig. 7). 


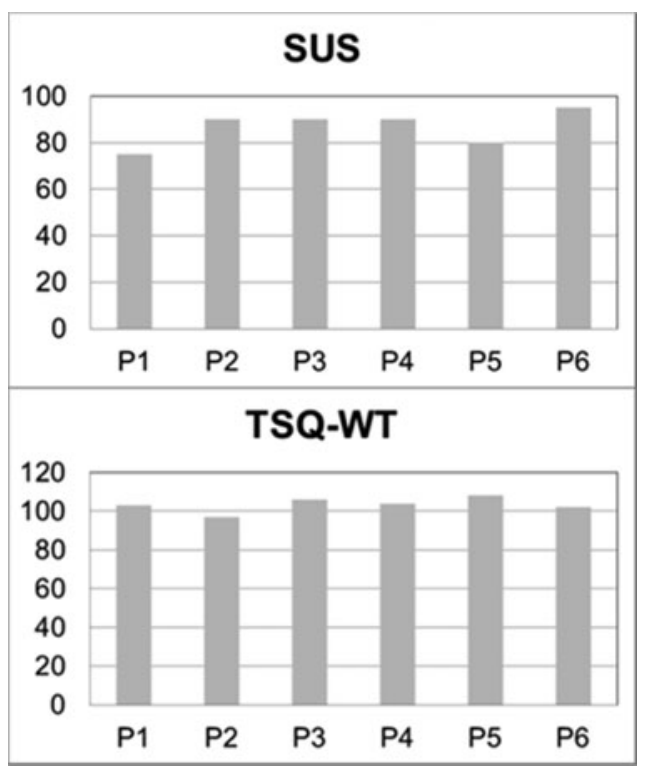

Fig. 7. Results of the evaluation of the user satisfaction and usability of the system in terms of system usability score (SUS) and Tele-healthcare Satisfaction Questionnaire - Wearable Technology (TSQ-WT).

This study presented some limitations. First, the results should be interpreted with caution since they could be affected by the small sample size and cannot be directly generalized to a similar population. Second, the weight compensation was manually fixed at the beginning of the session and was not modulated during the execution of the task. However, the torques required at the shoulder and elbow joint change during the movement, and thus an adaptive non-linear control of the weight compensation should be designed. ${ }^{6,7}$ The system was tested in a rehabilitation centre with specialized caregivers. However, to provide a complete evaluation of the system usability further tests should be performed in a home environment. Finally, being a passive exoskeleton, the here-proposed system can be exploited only by subjects who preserved some residual motor capabilities. However, the exoskeleton proposed here is part of a more complex system including several other modules, such as neuromuscular electrical stimulation to provide arm and hand motions, a motorized hand orthosis for grasping cylindrical objects, an eye tracking system or a P300 brain computer interface to capture the user's intention, that can be added to the exoskeleton so as to make the whole system useful also for more severe end-users. ${ }^{18}$

This preliminary evaluation boosts a further development of the here-proposed system towards a real use in a home environment (e.g. automatic setting of weight compensation, adjustment of weight compensation during arm movements, development of a left-arm system).

\section{Conclusions}

A passive robotic exoskeleton arm to support activities of daily life was developed and the functional and usability assessment of this system was evaluated on six end-users affected by neuro-motor disorders. All of the end-users were able to accomplish functional tasks, such as drinking or reaching, from the first testing day. Three out of six end-users even achieved an overall motor performance similar to a group of healthy subjects (overall score $<4$ ). An overall high level of usability and user satisfaction was highlighted, suggesting that an $\mathrm{AD}$ based on a patient-cooperative approach very positively impacts on social inclusion, self-esteem and autonomy.

\section{Acknowledgements}

This work was supported by the European Project MUNDUS (FP7 ICT 2009-4; grant agreement no.: 248326). We would like to thank all the partners of the MUNDUS consortium who strongly 
collaborated in the development of the system, and all the people who agreed to participate in the experimental trials.

\section{References}

1. J. D. Ripat and R. L. Woodgate, "The role of assistive technology in self-perceived participation," Int. J. Rehabil. Res. 35, 170-177 (2012).

2. S. W. Brose, D. J. Weber, B. A. Salatin, G. G. Grindle, H. Wang, J. J. Vazquez and R. A. Cooper, "The role of assistive robotics in the lives of persons with disability," Am. J. Phys. Med. Rehabil. Assoc. Acad. Physiatr. 89, 509-521 (2010).

3. V. Maheu, J. Frappier, P. S. Archambault and F. Routhier, "Evaluation of the JACO robotic arm: Clinicoeconomic study for powered wheelchair users with upper-extremity disabilities," Proceedings of the 2011 IEEE International Conference Rehabilitation Robotics, Zurich, Switzerland (Jun. 29-Jul. 1, 2011) pp. $1-5$.

4. B. J. Driessen, H. G. Evers and J. A. van Woerden, "MANUS-a wheelchair-mounted rehabilitation robot," Proc. Inst. Mech. Eng. [H] 215, 285-290 (2001).

5. Z. Bien, D.-J. Kim, M.-J. Chung, D.-S. Kwon and P.-H. Chang, "Development of a wheelchair-based rehabilitation robotic system (KARES II) with various human-robot interaction interfaces for the disabled," Proceedings of the 2003 IEEE/ASME International Conference on Advanced Intelligent Mechatronics, Kobe, Japan (Jul. 20-24, 2003), vol. 2, pp. 902-907.

6. B. T. Iwamuro, E. G. Cruz, L. L. Connelly, H. C. Fischer and D. G. Kamper, "Effect of a gravitycompensating orthosis on reaching after stroke: evaluation of the Therapy Assistant WREX," Arch. Phys. Med. Rehabil. 89, 2121-2128 (2008).

7. T. Rahman, W. Sample, S. Jayakumar, M. M. King, J. Y. Wee, R. Seliktar, M. Alexander, M. Scavina and A. Clark, "Passive exoskeletons for assisting limb movement," J. Rehabil. Res. Dev. 43, 583-590 (2006).

8. J. L. Herder, N. Vrijlandt, T. Antonides, M. Cloosterman and P. L. Mastenbroek, "Principle and design of a mobile arm support for people with muscular weakness," J. Rehabil. Res. Dev. 43, 591-604 (2006).

9. T. Nef, M. Mihelj and R. Riener, "ARMin: a robot for patient-cooperative arm therapy," Med. Biol. Eng. Comput. 45, 887-900 (2007).

10. J. Nikitczuk, B. Weinberg and C. Mavroidis, "Control of electro-rheological fluid based resistive torque elements for use in active rehabilitation devices," Smart Mater. Struct. 16, 418 (2007).

11. H. I. Krebs, N. Hogan, M. L. Aisen and B. T. Volpe, "Robot-aided neurorehabilitation," IEEE Trans. Rehabil. Eng. 6, 75-87 (1998).

12. V. Klamroth-Marganska, J. Blanco, K. Campen, A. Curt, V. Dietz, T. Ettlin, M. Felder, B. Fellinghauer, M. Guidali et al., "Three-dimensional, task-specific robot therapy of the arm after stroke: a multicentre, parallel-group randomised trial," Lancet Neurol. 13, 159-166 (2014).

13. K. Kiguchi and Y. Hayashi, "An EMG-based control for an upper-limb power-assist exoskeleton robot," IEEE Trans. Syst. Man Cybern. B 42, 1064-1071 (2012).

14. T. Lenzi, S. M. M. De Rossi, N. Vitiello and M. C. Carrozza, "Intention-based EMG control for powered exoskeletons," IEEE Trans. Biomed. Eng. 59, 2180-2190 (2012).

15. M. Casadio, V. Sanguineti, C. Solaro and P. G. Morasso, "A haptic robot reveals the adaptation capability of individuals with multiple sclerosis.," 1225-1233 (2007).

16. P. S. Lum, C. G. Burgar and P. C. Shor, "Evidence for improved muscle activation patterns after retraining of reaching movements with the MIME robotic system in subjects with post-stroke hemiparesis," IEEE Trans. Neural Syst. Rehabil. Eng. 12, 186-194 (2004).

17. H. Kim, L. M. Miller, I. Fedulow, M. Simkins, G. M. Abrams, N. Byl and J. Rosen, "Kinematic data analysis for post-stroke patients following bilateral versus unilateral rehabilitation with an upper limb wearable robotic system," IEEE Trans. Neural Syst. Rehabil. Eng. 21, 153-164 (2013).

18. A. Pedrocchi, S. Ferrante, E. Ambrosini, M. Gandolla, C. Casellato, T. Schauer, C. Klauer, J. Pascual, C. Vidaurre et al., "MUNDUS project: MUltimodal Neuroprosthesis for daily Upper limb Support," J. Neuroengineering Rehabil. 10, 66 (2013).

19. M. Rohm, M. Schneiders, C. Müller, A. Kreilinger, V. Kaiser, G. R. Müller-Putz and R. Rupp, "Hybrid brain-computer interfaces and hybrid neuroprostheses for restoration of upper limb functions in individuals with high-level spinal cord injury," Artif. Intell. Med. 59, 133-142 (2013).

20. E. Ambrosini, S. Ferrante, T. Schauer, C. Klauer, M. Gaffuri, G. Ferrigno and A. Pedrocchi, "A myocontrolled neuroprosthesis integrated with a passive exoskeleton to support upper limb activities," J. Electromyogr. Kinesiol. 24, 307-317 (2014).

21. W. Reichenfelser, J Karner and M. Gföhler, "Design concept for a mobile arm support," Proceedings of the $20^{\text {th }}$ Annual Meeting of the Europe Society of Movement Analysis for Adults and Children, Vienna, Austria (Sep. 12-17, 2011) pp. 162-163.

22. E. Ambrosini, S. Ferrante, M. Gföhler, W. Reichenfelser, J. Karner, T. Schauer, C. Klauer, G. Ferrigno and A. Pedrocchi, "A hybrid assistive system to support daily upper limb activities," Proceedings of the $17^{\text {th }}$ Annual International FES Society Conference, Banff, Canada (Sep. 9-12, 2012) pp. 1-4.

23. M. Gilliaux, T. Lejeune, C. Detrembleur, J. Sapin, B. Dehez and G. Stoquart, "A robotic device as a sensitive quantitative tool to assess upper limb impairments in stroke patients: A preliminary prospective cohort study," J. Rehabil. Med. 44, 210-217 (2012). 
24. V. P. Stokes, H. Lanshammar and A. Thorstensson, "Dominant pattern extraction from 3-D kinematic data," IEEE Trans. Biomed. Eng. 46, 100-106 (1999).

25. G. Baroni, A. Pedrocchi, G. Ferrigno, J. Massion and A. Pedotti, "Motor coordination in weightless conditions revealed by long-term microgravity adaptation," Acta Astronaut. 49, 199-213 (2001).

26. J. Brooke, "SUS: A ‘Quick and Dirty' Usability Scale," In: Usability Evaluation in Industry (P. W. Jordan, B. Thomas, B. A. Weerdmeester and A. L. McClelland, eds.) (Taylor and Francis, London, 1996) pp. 1-7.

27. L. A. Corben, N. Georgiou-Karistianis, J. L. Bradshaw, D. R. Hocking, A. J. Churchyard and M. B. Delatycki, "The Fitts task reveals impairments in planning and online control of movement in Friedreich ataxia: Reduced cerebellar-cortico connectivity?,"Neuroscience 192, 382-390 (2011).

28. L. A. Corben, M. B. Delatycki, J. L. Bradshaw, M. K. Horne, M. C. Fahey, A. J. Churchyard and N. Georgiou-Karistianis, "Impairment in motor reprogramming in Friedreich ataxia reflecting possible cerebellar dysfunction,” J. Neurol. 257, 782-791 (2010). 